\title{
Re: Epigenetics of Cellular Reprogramming
}

\author{
Krishnakumar R, Blelloch RH \\ Eli and Edythe Broad Center of Regeneration Medicine and Stem Cell Research, Center for Reproductive Sciences and Clinic of Urology, \\ University of California San Francisco, California, USA
}

Curr Opin Genet Dev 2013;23:548-555.

\section{EDITORIAL COMMENT}

Cells have some specific molecular and physiological properties that act their functional process. However, many cells have an ability of efficient transition from one type to another. This ability is named plasticity. This process occurs due to epigenetic reprogramming that involves changes in transcription and chromatin structure. Some changes during reprogramming that have been identified in recent years as genomic demethylation (both histone and DNA), histone acetylation and loss of heterochromatin during the development of many diseases such as infertility and cancer progression. In this review, the authors focused on the latest work addressing the mechanisms surrounding the epigenetic regulation of various types of reprogramming, including somatic cell nuclear transfer, cell fusion and transcription factor- and microRNA-induced pluripotency. There are many responsible factors such as genes, cytokines, proteins, co-factors (i.e. vitamin C) in this local area network. The exact mechanisms by which these changes are achieved and the detailed interplay between the players responsible, however, remain relatively unclear. In the treatment of diseases, such as infertility, urooncology, reconstructive urology, etc., epigenetic changes and cellular reprogramming will be crucial in the near future. Central to achieving that goal is a more thorough understanding of the epigenetic state of fully reprogrammed cells. By the progress of researches on this topic, new treatment modalities will be identified for these diseases.

Fehmi Narter, MD

\section{Re: Prospective Pilot Study of Mirabegron in Pediatric Patients with Overactive Bladder}

\section{Blais AS, Nadeau G, Moore K, Genois L, Bolduc S}

CHU de Québec-Université Laval, Department of Urology, Québec, Canada

Eur Urol 2016;70:9-13. doi: 10.1016/j.eururo.2016.02.007. Epub 2016 Feb 11.

\section{EDITORIAL COMMENT}

Overactive bladder $(\mathrm{OAB})$ is a highly prevalent disorder in the pediatric population. This event negatively affects and impairs children's development. Antimuscarinics are the mainstay pharmacological management of $O A B$, but their side effects profile limits their use. Mirabegron, a new molecule with a distinct mechanism of action (b3-adrenoreceptor agonist), was recently approved as monotherapy for idiopathic OAB in adults but has not been studied in the pediatric population. Blais et al. have evaluated the efficacy and safety of mirabegron in the treatment of urinary incontinence in children with idiopathic $0 A B$ who were refractory to and/or intolerant of antimuscarinics. This prospective off-label study, using an adjusted-dose regimen of mirabegron (25-50 mg), included pediatric patients with refractory urinary incontinence due to OAB. Patients without symptom improvement or with partial response under intensive behavioral protocol and medical therapy (at least two different antimuscarinic agents) were included in the study. Their primary outcome was better reported efficacy than with the use of prior anticholinergic medication. Secondary end points were tolerability and safety. Families were questioned for continence, side effects, compliance, and Patient Perception of Bladder Condition (PPBC) questionnaire. A total of 58 patients (14 girls, 44 boys) were enrolled in a mirabegron open-label trial. The median age at initiation of mirabegron was 10.1 years [interquartile range (IQR): 8.8-13.5]. The median duration of treatment was 11.5 months (IQR: 6.0-15.0). The median bladder capacity improved from $150 \mathrm{ml}$ to $200 \mathrm{ml}(\mathrm{p}<0.001)$. Continence improved in 52 of 58, with 13 being completely dry. The median PPBC score improved from 4.0 to 2.0 ( $p<0.001)$. The authors also assessed the safety of mirabegron. All electrocardiogram and blood tests remained normal. No significant change in blood pressure $(<5 \mathrm{mmHg})$ and heart rate (fewer than five beats per minute) was reported. Fifty patients (86\%) did not report any side effects. A subgroup of 5 (9\%) described mild side effects i.e. transient abdominal colic (2), constipation (2), and blurred vision (1). Three patients, however, (5\%) discontinued the treatment because of side effects; one patient had significant nasopharyngitis, one patient had nausea, and one had a change in behavior (as if his attention deficit hyperactivity disorder treatment was no longer effective). As a result, mirabegron, a novel first-in-class therapy, appears to be a safe alternative for children with idiopathic $O A B$ and allows improvement of symptoms in patients refractory to antimuscarinics. Absence of a placebo group was a limitation of the study. However, patients had already failed two trials of medication, decreasing the likelihood of a placebo effect. 